\title{
Maximum total welfare versus growth of R\&D networks
}

Mohamad Alghamdi*

\section{"Correspondence:}

almohamad@ksu.edu.sa

Department of Mathematics,

College of Sciences, King Saud

University, P.O. Box 2455, Riyadh,

11451, Saudi Arabia

\section{Springer}

\begin{abstract}
The aim of this paper is to explore the characteristics of symmetric R\&D networks that generate the highest overall welfare in a setting where spillovers between non-cooperating firms are ignored. The relationship between the network population and the outcomes is shown to be sensitive to the market structure. The first result pertains to the features of the successive efficient networks. In a differentiated product market, the consistency of the social and individual benefits is not affected by expanding the network. In contrast, the social benefit in a homogeneous product market limits the cooperation between firms. This in turn creates a gap between individual and social profits (an undesirable area). This gap between the two perspectives is maximized by increasing the network size. The second result concerns the expected improvement in the total welfare with respect to the network size. The results show that the benefit behind expanding the socially optimal structure is maximized when firms belong to a differentiated product market.
\end{abstract}

Keywords: symmetric networks; network size; equilibria; welfare maximization

\section{Introduction}

We consider a network game for firms conduct R\&D to reduce the cost of the production. In an R\&D network, the players (firms) are represented by nodes and the R\&D partnerships are represented by links. ${ }^{\text {a }}$ The network game consists of three stages: network formation, $R \& D$ investment (effort) and market competition. The R\&D effective efforts of firms equal to own efforts and partial efforts of other firms in a network determined by an R\&D spillover. Technically, if any two firms are linked, the spillover is set one; otherwise there is a free spillover less than one.

Connecting R\&D theory with the network concepts addresses substantial objectives in the R\&D literature (e.g., [1-4]). It contributes to understanding the effects of R\&D agreements (cooperative links) on expenditures by firms. The network approach exhibits several categories of R\&D partnerships, so that different structures of collaborative networks can be described. From these networks there are individually and socially desirable networks that can be examined by using stability and efficiency conditions. Stability of networks provides a preferable structure for firms to gain higher profits. Efficiency of networks matches the desires of producers and of consumers in an optimal structure. One important aspect of this issue is that the stable network is not always efficient. For symmetric networks (each firm has the same number of links) with arbitrary firms producing homogeneous goods

(c) The Author(s) 2017. This article is distributed under the terms of the Creative Commons Attribution 4.0 International License (http://creativecommons.org/licenses/by/4.0/), which permits unrestricted use, distribution, and reproduction in any medium, provided you give appropriate credit to the original author(s) and the source, provide a link to the Creative Commons license, and indicate if changes were made. 
and competing by their quantities, Goyal and Moraga-Gonzalez [3] found that complete networks are stable, but not efficient.

In this paper, we explore the characteristics of networks that yield the highest possible total welfare. We consider the symmetric (regular) networks with an arbitrary number of firms. The reason for taking this type of networks is in difficulty study the efficiency with large number of firms where then we need to consider a large number of different networks and extract a large set of equilibria.

In particular, we use the R\&D network model by Goyal and Moraga-Gonzalez to answer the following questions with a liner-quadratic utility function under Cournot competition for independent and homogeneous goods:

(1) How might increase of competitors wide the gap between the stability and efficiency of R\&D network?

(2) What are the characteristics of efficient networks?

(3) What is the density of the efficient network if the network size increases?

The results of this paper can be summarized as follows. The first result concerns the relationship between characteristics of the efficient networks with a growing number of firms. In a setting where goods are independent, for all network sizes the complete network is uniquely efficient. ${ }^{\mathrm{b}}$ If goods are homogeneous, there is a range of network sizes that are maximized at the same activity level. For an even size, the sizes followed thereafter that size are maximized at the same activity level. This allows the efficiency with a growing number of firms forms stairs (a stair function).

The second result compares the structure of the efficient network with the strategically stable network. If goods are independent, the original results by Goyal and MoragaGonzalez are not affected by growing the network size. For homogeneous goods, first, we observe that, the gap between efficiency and stability increases with growing the network size. Second, the density of the efficient network increases and decreases with growing network size.

The third result compares the activity levels at which the consumer surplus, industry profit and welfare are maximized and this comparison is with the expansion of the network. For independent goods, these equilibria are maximized at the same activity level. However, for homogeneous goods, consumer surplus, industry profit and the welfare are maximized at different activity levels. In general, the level that maximizes is higher than the other two levels. This result points out that the efficient network is not a complex network as the case for maximizing the industry profit.

In addition, when comparing the total welfare of the efficient networks of different sizes, it is found that the difference is not small if goods are independent. However, if the goods are homogeneous, the difference is small despite of the large number of firms or activity levels. This result for homogeneous goods indicates that the social incentive for firms to form new $R \& D$ agreements could be weak.

The intuition underlying these findings is mainly derived from linking R\&D model to the network concept. Newcomers into the network may not be a necessary point to increase the $R \& D$ agreements in order to maximize the social return. Also, the individual incentives and overall economic welfare do not always match and the gradual and regular construction may increase the gap between them.

The remainder of the article is organized as follows. In the second section, we provide foundations in the social network and microeconomics and we introduce the Goyal and 
Moraga-Gonzalez model. In the third section, we present our outcomes. In the fourth section, we conclude our study.

\section{Method}

\subsection{Network}

A network is formed by a set of vertices (nodes) and a set of edges (links) connecting these vertices $[5,6]$. We define $N$ as a set of all vertices labeled by letters $\{i, j, k, \ldots\}$ where $|N|=n$ and $E=\{i j, j k, \ldots\}$ is a set of all edges in the network where $|E|=m$ is the number of links. Then $G(N, E)$ denotes a network with nodes $N$ and links $E$, and for simplicity the network is denoted by $G$. We focus on undirected networks; meaning that each link between any two vertices runs in both directions. Thus, each two links $i j$ and $j i$ in network $G$ are the same. We also focus on simple networks where there are no parallel edges (edges that have the same end vertices) or loops (edges where their start and end vertices are the same).

Nodes linked to node $i \in N$ is defined as a set of neighbors of that node: $N_{i}=\{j \in N: i j \in$ $E$ \}. The length of the neighbors' set of node $i$ is a degree of that node. Thus, the degree of each node $i \in N$ is denoted by $\operatorname{deg}(i)=\left|N_{i}\right|$ where $0 \leq \operatorname{deg}(i) \leq n-1$.

The density of network $G$ gives a ratio of actual links in the network out of possible links that can be drawn with the same number of nodes $D(G)=2 m /(n(n-1))$. Note that, the density of network $G$ belongs to the interval $[0,1]$ where if $D(G)=0$, the network $G$ is an empty network (no links between nodes); whereas if $D(G)=1$, the network $G$ is a complete network (each two nodes are linked).

In this paper. we consider symmetric networks (regular network). The $k$-regular network is a graph such that each node has the same number of links, $k$. Note that the complete network is also defined as a $(n-1)$-regular network because each node has $n-1$ links and the cycle network is a 2-regular network since each node has two links. For simplicity, the network $\mathcal{G}_{n}$ denotes to a symmetric network consisted of $n$ nodes.

\subsection{The model}

The emphasis in this paper is on the linear-quadratic function of consumers given by [7]:

$$
U=a \sum_{i=1}^{n} q_{i}-\frac{1}{2}\left(\alpha \sum_{i=1}^{n} q_{i}^{2}+2 \lambda \sum_{j \neq i} q_{i} q_{j}\right)+I .
$$

Here the demand parameters $a>0$ denotes the willingness of consumers to pay and $\alpha>0$ is the diminishing marginal rate of consumption, while $q_{i}$ is the quantity consumed of good $i$ and $I$ measures the consumer's consumption of all other products. Without loss of generality, it is assumed that $\alpha=1$ to simplify the analysis. The parameter $\lambda$ such that $-1 \leq \lambda \leq 1$ captures the marginal rate of substitution between different products.

Let $m$ is a consumer's income and $p_{i}$ is the price of good $i$. If the consumer buys $q_{i}$ of good $i$, the money spent is $p_{i} q_{i}$ where $\sum p_{i} q_{i}+I \leq m$ (budget constraint). The balance after consuming the good $i$ is $I=m-p_{i} q_{i}$ and by substituting into the equation (1), the result is

$$
U=a \sum_{i=1}^{n} q_{i}-\frac{1}{2}\left(\sum_{i=1}^{n} q_{i}^{2}+2 \lambda \sum_{j \neq i} q_{i} q_{j}\right)+m-p_{i} q_{i} .
$$


The utility function (1) is a concave function, so the first order condition $\left(\frac{\partial U}{\partial q_{i}}=0\right)$ determines the optimal consumption of $\operatorname{good} i$. Thus $\frac{\partial U}{\partial q_{i}}=a-q_{i}-\lambda \sum_{j \neq i} q_{j}-p_{i}=0$ and this means that the inverse demand function for each good $i, D_{i}^{-1}$ is

$$
D_{i}^{-1}=p_{i}=a-q_{i}-\lambda \sum_{j \neq i} q_{j}, \quad i=1, \ldots, n
$$

The profit $\pi_{i}$ for firm $i$ is

$$
\pi_{i}=\left(p_{i}-c_{i}\right) q_{i}=\left(a-q_{i}-\lambda \sum_{j \neq i}^{n} q_{j}-c_{i}\right) q_{i}
$$

where $p_{i}$ is the price of good $i$ produced by firm $i$ and $c_{i}$ is the production cost.

The consumer surplus is the difference between the price that consumers are willing to pay for a product and the actual market price. The consumer surplus is expressed as

$$
C S=\frac{(1-\lambda)}{2} \sum_{i=1}^{n} q_{i}^{2}+\frac{\lambda}{2}\left(\sum_{i=1}^{n} q_{i}\right)^{2} .
$$

Industry surplus is defined as the sum of all profits made by all producers in the market: $\Pi=\sum_{i=1}^{n} \pi_{i}$. The total Welfare $(T W)$ is the total surplus of consumers and producers and is defined by

$$
T W=C S+\Pi
$$

\subsection{R\&D network model}

$R \& D$ cooperation between firms have been studied in terms of networks in many papers (e.g., $[1-3,8])$. The focus of this paper is on Goyal and Moraga-Gonzalez, who readdressed the R\&D cooperation model that was presented by D'Aspremont and Jacquemin [9] after using the networks. ${ }^{\mathrm{c}}$ In Goyal and Moraga-Gonzalez paper, if firms cooperate in R\&D, they are linked in an undirected network and spillover is set at one where the cost of link formation is assumed to be negligible. If firms do not cooperate, they are not linked and there is an identical spillover $(\beta \in[0,1))$ between non-linked firms.

- Stages of the model:

In Goyal and Moraga-Gonzalez, firms strategically form bilateral collaborative links with other firms where the collaboration of firms is modeled as a three-stage game.

The first stage: Each firm chooses its research partners. Firms and the cooperative links together constitute a network of cooperation in R\&D.

The second stage: Given the R\&D network, each firm chooses the amounts of investment (effort) in R\&D simultaneously and independently in order to reduce the cost of production.

The third stage: Given the R\&D investments of each firm and the effective R\&D effort (as determined by the R\&D network), firms compete in the product market by setting quantities (Cournot competition) in order to maximize their profits. 


\section{- Cost reduction:}

In Goyal and Moraga-Gonzalez, the effective R\&D effort for each firm is defined by the following equation:

$$
X_{i}=x_{i}+\sum_{j \in N_{i}} x_{j}+\beta \sum_{k \notin N_{i}} x_{k}, \quad i=1, \ldots, n,
$$

where $x_{i}$ denotes R\&D effort of firm $i, N_{i}$ is the set of firms participating in a joint venture with firm $i$ and $\beta \in[0,1)$ is an exogenous parameter that captures knowledge spillovers acquired from firms not engaged in a joint venture with firm $i$. The effective R\&D effort reduces firm $i$ 's marginal cost $(\bar{c})$ of production

$$
c_{i}=\bar{c}-x_{i}-\sum_{j \in N_{i}} x_{j}-\beta \sum_{k \notin N_{i}} x_{k}, \quad i=1, \ldots, n .
$$

The effort is assumed to be costly and the function of the cost is quadratic, so that the cost of R\&D is $\gamma x_{i}^{2}$, where $\gamma>0$ indicates the effectiveness of R\&D expenditure [9]. The profit $\pi_{i}$ for firm $i$ is the difference between revenue and production cost minus the cost of R\&D

$$
\pi_{i}=\left(a-\sum_{i=1}^{n} q_{i}-\bar{c}+x_{i}+\sum_{j \in N_{i}} x_{j}+\beta \sum_{k \notin N_{i}} x_{k}\right) q_{i}-\gamma x_{i}^{2}, \quad i=1, \ldots, n,
$$

where the marginal cost satisfy $a>\bar{c}$. From the last equation, we can find the best response function of R\&D effort for each firm $i$ by calculating the first order condition $\left(\frac{\partial \pi_{i}}{\partial x_{i}}=0\right)$.

- RED network:

In an asymmetric R\&D network, the distribution of links between firms is heterogeneous. However, in a symmetric R\&D network, each firm has the same number of cooperative links (neighbors). If $\mathcal{G}$ is a symmetric network of degree $k$, this means each firm has $k$ links (cooperative activity levels). Figure 1 presents some examples of symmetric networks of size five firms.

- The equilibria for independent and homogeneous goods:

For symmetric networks, we assume that the R\&D spillover is set zero $(\beta=0)$. Thus, the effective effort function for each firm $i$ becomes

$$
X_{i}=x_{i}+\sum_{j \in N_{i}} x_{j}, \quad i=1, \ldots, n
$$

The equilibria under Cournot competition for symmetric networks are cited form Goyal and Moraga-Gonzalez paper (listed in the Appendix).

Figure 1 Examples of symmetric networks of size four firms.

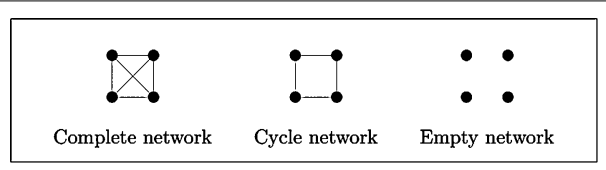




\section{- The effectiveness $\gamma$ :}

According to Goyal and Moraga-Gonzalez, if goods are independent, then the effectiveness should satisfy

$$
\begin{aligned}
& \gamma>\max \{a n / 4 \bar{c}, n / 4\} \quad \text { if } \lambda=0, \\
& \gamma>\max \left\{n^{2} /(n+1)^{2}, a / 4 \bar{c}\right\} \quad \text { if } \lambda=1 .
\end{aligned}
$$

\section{- Stability and efficiency of networks:}

Linking R\&D cooperation of firms to the network concepts involves the concepts of pairwise stability and efficiency. The definition of the network's pairwise stability depends on firms' profit functions and it is a necessary condition for strategic stability as shown in [10].

Definition 1 (Pairwise Stability) For any network $G$ to be stable, the following two conditions need to be satisfied for any two firms $i, j \in G$ :

1. If $i j \in G, \pi_{i}(G) \geq \pi_{i}(G-i j)$ and $\pi_{j}(G) \geq \pi_{j}(G-i j)$.

2. If $i j \notin G$ and if $\pi_{i}(G)<\pi_{i}(G+i j)$, then $\pi_{j}(G)>\pi_{j}(G+i j)$.

$G-i j$ is the network resulting from deleting a link $i j$ from the network $G$ and $G+i j$ is the network resulting from adding a link $i j$ to the network $G$. From this definition, network $G$ is stable if no firm can obtain higher profit from deleting one of its links; and any other link between two firms would benefit only one of them.

The definition of the efficiency of a network that is given as follows and is determined by the total welfare generated from that network.

Definition 2 (Network Efficiency) Network $G$ is said to be efficient if no other network Ǵ can be generated by adding or deleting links, such that $T W(G)>T W(G)$.

\section{Results: structure of the efficient symmetric networks}

\subsection{Fixed size of the network}

Goyal and Moraga-Gonzalez [3] examined an oligopolistic market under Cournot competition with linear demand for two types of $R \& D$ networks in terms of the degree distribution. In the first case, the collaborative activity is symmetrically distributed between an arbitrary number of firms where the spillover term is set at zero. In the second case, the collaborative activity is asymmetrically distributed between few firms where the spillover term is involved.

Concerning the symmetric networks, Goyal and Moraga-Gonzalez studied the effect of the growth of the activity levels (cooperative links) on the equilibrium outcomes for independent and homogeneous goods. The main findings show the role of the products type in determining the benefit of establishing the activity levels. If goods are independent, the equilibrium outcomes increase with growing the cooperative activity level. In terms of the stability and efficiency of the R\&D network, this result indicates that the complete network is uniquely stable and efficient.

However, if goods are homogeneous, the effect of the activity level on the equilibrium outcomes is different. While R\&D effort decreases as the activity level increases, the industry profit and total welfare are maximized at different intermediate levels of the cooperative activity. This indicates that the complete and the empty networks are not efficient. 
For the stability of the R\&D network, they stated that the complete network is a stable network.

\subsection{Growing size of the network}

In this section, we examine the effect of expanding the population of the R\&D network on the total welfare. The concern is on the R\&D networks that generates highest possible total welfares and on determining the role of the market structure in developing the $R \& D$ partnerships. The results of our discussion are stated without proofs. This is because the efficient network for homogeneous goods cannot be characterized by an especial equation or by features that remain constant for any network size like the stability of the complete network. Instead, we provide examples to capture our outcomes.

According to the outcomes of Goyal and Moraga-Gonzalez, if goods are independent, the complete network is uniquely efficient and stable. We show that this result is not affected by growing the network size. This indicates that the fully connected network is a socially preferable structure regardless of the number of firms in the market.

However, if goods are homogeneous, the total welfare of the socially optimal structure has different behavior. When plotting the total welfare as a function of the number of firms, the result forms stairs (a stair function). The reason of appearing the total welfare in this shape is that a symmetric network is not existed for any network size $n$ and activity level $k$. For example, if $n$ and $k_{\max }$ that maximizes the total welfare are odd, the symmetric network cannot be established. Therefore, we have to look for an even activity level $k$ such that the total welfare is maximized.

Proposition 1 Given a symmetric network structure $\mathcal{G}_{n}$ with zero spillover such that the effectiveness $\gamma$ satisfies (10). Let $T W\left(\mathcal{G}^{*}{ }_{n}\right)$ denotes the economic welfares from efficient patterns $\mathcal{G}^{*}{ }_{n}$ where $n$ is even. If goods are homogeneous, the welfares $T W\left(\mathcal{G}^{*}{ }_{n+1}\right), T W\left(\mathcal{G}^{*}{ }_{n+2}\right)$ and $T W\left(\mathcal{G}^{*}{ }_{n+3}\right)$ are maximized at the same activity level $k_{\max }$.

Figure 2 shows the efficient networks under Cournot competition for homogeneous goods. When increasing the size of the $R \& D$ network, there is a slight increase in the activity level $k_{\max }$.

In addition, Goyal and Moraga-Gonzalez stated that the complete network is stable irrespective of the production type. However, the uniqueness of the stability of the complete network cannot be shown for homogeneous goods. This means that for this type of

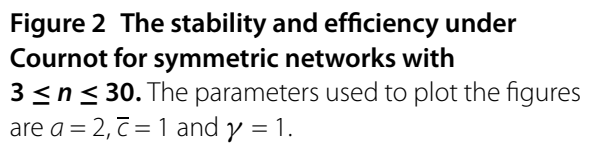


goods, it may exist other stable networks. For example, the authors found that for asymmetric networks in a market consisted of three firms, the partial network (the network $G_{4}$ in Figure 4) is also stable for a small value of the $R \& D$ spillover $\beta$.

In the following discussion, we compare the outcomes of the complete network with the socially optimal structure for two reasons. The first reason is that the complete R\&D network is stable in many papers (e.g., $[2-4,11])$. The second reason is that in the empirical $R \& D$ literature, several researchers have found that the interaction of firms in $R \& D$ exhibits characteristic features of complex networks (e.g., [12-15]). This indicates that firms in a real network always seek to establish new $R \& D$ agreements.

Concerning homogeneous goods, there exists a gap or an area between the stability and efficiency of the R\&D cooperation network. We refer to this area as an undesirable area in terms of the individual and social perspective. This gap is shown in Figure 2 for $3 \leq$ $n \leq 30$. The figure displays the activity levels at which the R\&D network becomes stable and efficient under homogeneous Cournot. ${ }^{\mathrm{d}}$ It can be observed that as the network size increases, the gap between the stability and efficiency increases.

This result can be shown again by examining the density of the stable and efficient networks. Figure 3 shows the gap between the stable and efficient networks for different sizes of the symmetric networks. As can be observed from the figure by increasing the number of firms in the R\&D network, the density of the efficient network increases and decreases. This reflects the positive and negative relationships between the density and the total welfare of the socially optimal structure. We also can observe that the fluctuation in the density of the efficient network reduces as the network size increases. This indicates that the gap between the stability and efficiency becomes almost constant as the cooperators increases in the network.

Another point can be extracted from Figure 3 is that for the symmetric networks, we might be able to determine an upper bound of the density of the efficient network. We can note that with increasing the network size, the density of the efficient network is less than 0.6. Also, if $\mathcal{G}^{*}{ }_{n}$ denotes the efficient patterns, we can expect that $\lim _{n \rightarrow \infty} D\left(\mathcal{G}^{*}{ }_{n}\right) \neq 0$; meaning that the efficient network will never become an empty network for any network size $n$. The last observation is not true if we consider an R\&D spillover between non-linked firms where Goyal and Moraga-Gonzalez found that with three firms produce homogeneous goods, the empty network is efficient if the spillover reaches its maximum values i.e., $\beta \rightarrow 1$.

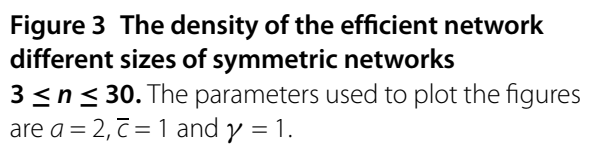


Figure 4 The asymmetric networks with three firms.

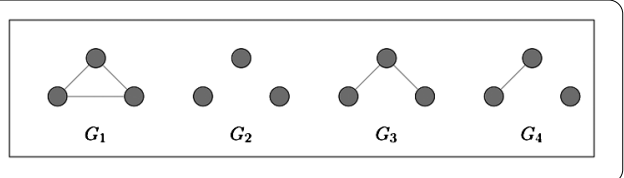

Figure 5 The asymmetric networks with four firms.

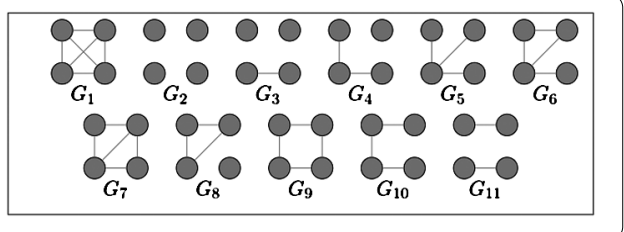

Figure 6 The total welfare of asymmetric networks given in Figures 4 and 5 . The parameters used to plot the figures are $a=2, \bar{c}=1$ and $\gamma=1$ if $n=3$ and $\gamma=2$ if $n=4$.

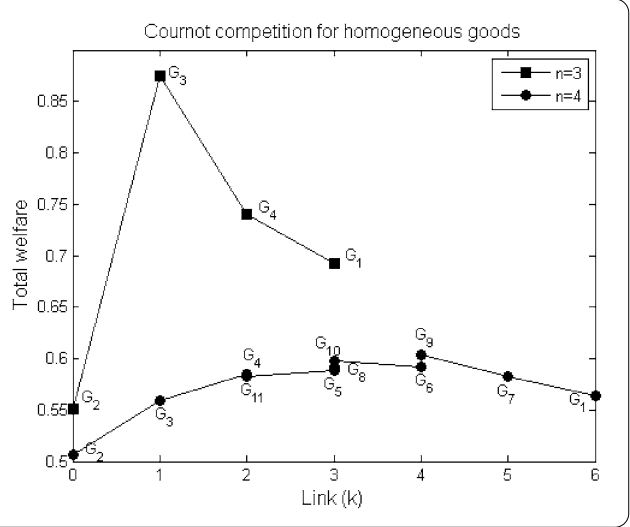

Proposition 2 Given a symmetric network structure $\mathcal{G}_{n}$ with zero spillover such that the effectiveness $\gamma$ satisfies (10). With respect to network size $n$,

1. the density of the efficient network $\mathcal{G}^{*}{ }_{n}$ increases if goods are independent;

2. the density of the efficient network $\mathcal{G}^{*}{ }_{n}$ fluctuates if goods are homogeneous.

Figures 4 and 5 show the list of asymmetric networks with three and four firms, respectively. Figure 6 shows the total welfare of those networks with respect to the activity level $k$ with zero spillover $(\beta=0)$. It can be observed that when there are three firms, the total welfare is maximized when there are two links (i.e., $G_{3}$ ) and when there are four firms, the total welfare is maximized when the cooperation forms a symmetric network with activity level $k=2$. When comparing these outcomes in asymmetric networks with those generated from symmetric networks, we find that the networks $\mathcal{G}_{3}$ and $\mathcal{G}_{4}$ are maximized at the activity level $k=2$ - see Figure 2 .

Now, from equation (5), the total welfare consists of two components: the consumer surplus and the industry profit. When comparing the activity levels that maximize the three functions are maximized, we find that for independent goods, the activity levels are identical. This result is straightforward from Proposition 2 since the total welfare increase with growing the density of the network. However, for homogeneous goods, the activity level that maximizes the industry profit is higher than the other activity levels that maximize the total welfare and the consumer surplus. 
Figure 7 The maximum total welfare, consumer surplus and industry profit for different sizes of symmetric networks $\mathbf{3} \leq \boldsymbol{n} \leq \mathbf{3 0}$. The parameters used to plot the figures are $a=2, \bar{c}=1$ and $\gamma=1$.

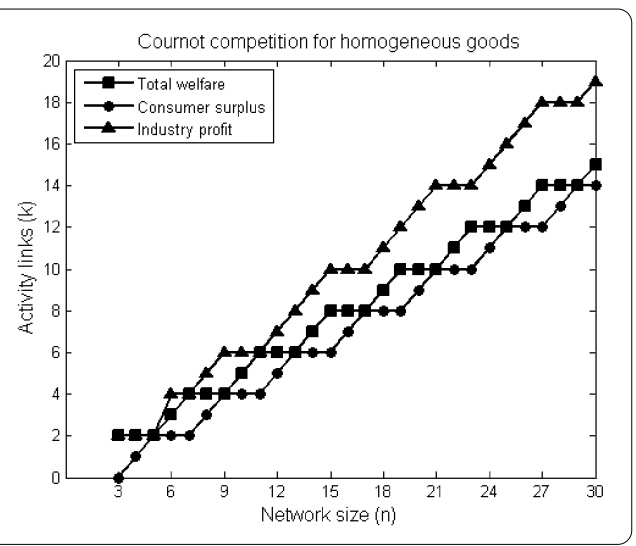

Proposition 3 Given a symmetric network structure $\mathcal{G}_{n}$ with zero spillover such that the effectiveness $\gamma$ satisfies (10). If the total welfare, consumer surplus and industry profit are maximized at $k_{T W}, k_{C S}$ and $k_{\Pi}$, respectively then with respect to the network size $n$,

1. $k_{\Pi}=k_{T W}=k_{C S}=n-1$ if goods are independent;

2. $k_{\Pi}>k_{T W} \geq k_{C S}$ if goods are homogeneous.

Figure 7 shows the activity levels at which the total welfare, consumer surplus and industry profit are maximized for different sizes of the symmetric networks.

In the following, we compare the total welfare of the socially optimal structure for different network sizes. When the determinant demand $a$ and the marginal cost $\bar{c}$ are fixed, the comparison shows that if goods are independent, the differences between the total welfares are not small. Since the complete network is the uniquely efficient network for independent goods, then for any network size $n$, the total welfare is

$$
T W\left(\mathcal{G}^{*}{ }_{n}\right)=\frac{n \gamma(6 \gamma-1)(a-\bar{c})}{(4 \gamma-n)^{2}} .
$$

By differentiating the total welfare with respect to the network size $n$, we have $d T W\left(\mathcal{G}^{*}{ }_{n}\right) /$ $d n=\gamma(6 \gamma-1)(4 \gamma+n) /(4 \gamma-n)^{3}$. Since $\gamma>n / 4$, then $d T W\left(\mathcal{G}^{*}{ }_{n}\right) / d n>0$ and this means that as the network size $n$ increases, the total welfare of the efficient network increases. Also, from equation (11), as the network size $n$ increases, the numerator increases and the denominator decreases, which in turn enlarges the gap between the total welfares of the efficient networks.

If the goods are homogeneous, the difference between the total welfares of the optimal networks is small compared to the case when the goods are independent. This result regarding to the total welfare as a function of the network size $n$ is not affected by growing the activity level $k$ as shown in Figure 8. This indicates that the maximum social benefit at $k_{\max }$ as the network size grows is high if goods are independent, but it might not be high if the goods are homogeneous.

Proposition 4 Given a symmetric network structure $\mathcal{G}_{n}$ with zero spillover such that the effectiveness $\gamma$ satisfies (10). If $\mathcal{G}^{*}{ }_{n}$ and $\mathcal{G}^{*}{ }_{n+1}$ are the successive efficient networks, then if goods are homogeneous, the difference $\left|T W\left(\mathcal{G}^{*}{ }_{n+1}\right)-T W\left(\mathcal{G}^{*}{ }_{n}\right)\right|$ is small compared to the case when the goods are independent. 
Figure 8 The welfare of the efficient networks. For $3 \leq n \leq 30$, the parameters used to plot the figures are $a=2, \bar{c}=1$ and $\gamma=1$ if goods are homogeneous and $\gamma=15$ if goods are independent.
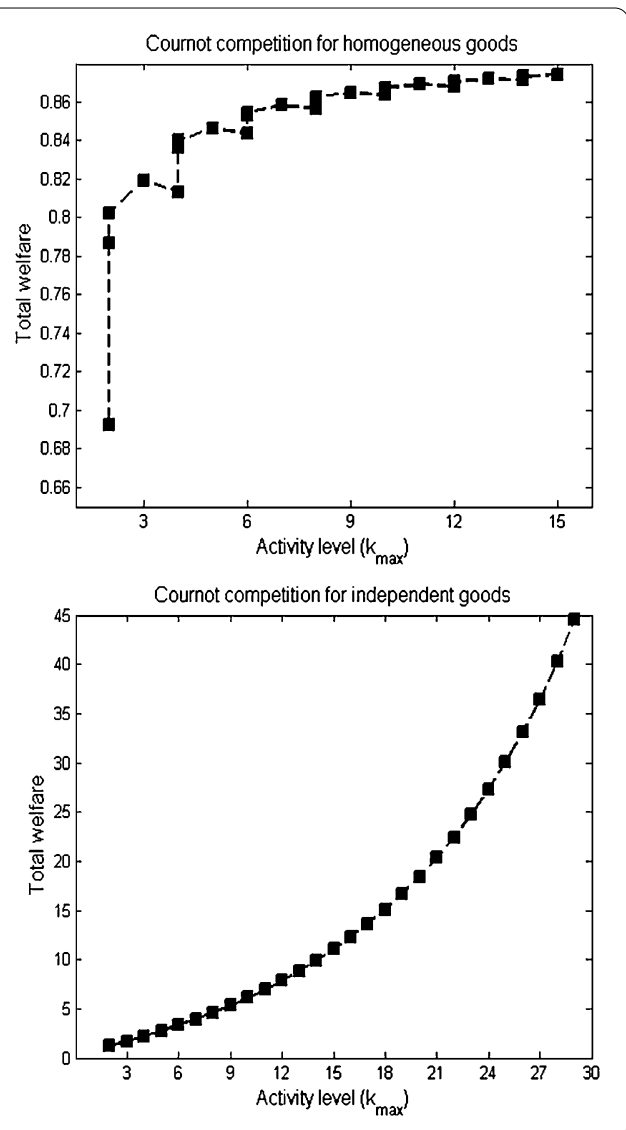

\section{Conclusion}

In this paper, we characterized the symmetric networks that yield the highest possible total welfare. Under Goyal and Moraga-Gonzalez model for independent and homogeneous goods, we examined the effect of expanding the socially optimal R\&D network on the outcomes.

For independent goods, the social benefit in the optimal structure is affected positively by growing the number of cooperators in $\mathrm{R} \& \mathrm{D}$. This indicates that the individual and social preferences in terms of forming $R \& D$ partnerships are consistent irrespective of the network size. However, for homogeneous goods, the social benefit limits the R\&D partnerships between firms. The outcomes show that the expansion of the network generates a gap between the individually and socially optimal structures. Moreover, increasing the network size does not provide a prominent progress in the total welfare compared to the benefit acquired if firms produce independent goods.

\section{Appendix}

- The equilibria under symmetric networks with an arbitrary number of firms $n$ :

For independent goods:

Effort: $x^{*}=\frac{(a-\bar{c})}{4 \gamma-k-1}$.

Quantity: $q^{*}=\frac{2 \gamma(a-\bar{c})}{4 \gamma-k-1}$.

Profit: $\pi^{*}=\frac{\gamma(4 \gamma-1)(a-\bar{c})^{2}}{(4 \gamma-k-1)^{2}}$.

Total welfare: $T W^{*}=\frac{n \gamma(6 \gamma-1)(a-\bar{c})^{2}}{(4 \gamma-k-1)^{2}}$. 
For homogeneous goods:

Effort: $x^{*}=\frac{(n-k)(a-\bar{c})}{\gamma(n+1)^{2}-(n-k)(k+1)}$.

Quantity: $q^{*}=\frac{\gamma(n+1)(a-\bar{c})}{\gamma(n+1)^{2}-(n-k)(k+1)}$.

Profit: $\pi^{*}=\frac{\left(\gamma^{2}(n+1)^{2}-(n-k)^{2}\right)(a-\bar{c})^{2}}{\left(\gamma(n+1)^{2}-(n-k)(k+1)\right)^{2}}$.

Total welfare: $T W^{*}=\frac{n\left(\gamma^{2}(n+2)(n+1)^{2}-2(n-k)^{2}\right)(a-\bar{c})^{2}}{2\left(\gamma(n+1)^{2}-(n-k)(k+1)\right)^{2}}$.

- The equilibria under asymmetric networks with three firms:

$$
\begin{aligned}
& x_{G_{1}}=\frac{(a-\bar{c})}{\left(\left(4 \lambda^{2}+8 \lambda+4\right) \gamma-3\right)}, \\
& q_{G_{1}}=\frac{(2 \gamma(\lambda+1)(a-\bar{c}))}{\left(\left(4 \lambda^{2}+8 \lambda+4\right) \gamma-3\right)}, \\
& x_{G_{2}}=\frac{(a-\bar{c})(\lambda(2 \beta-1)-2)}{2+4 \beta-8 \gamma+\left(1-12 \gamma-4 \beta^{2}\right) \lambda+4 \gamma \lambda^{3}} \text {, } \\
& q_{G_{2}}=\frac{\left(2 \gamma(a-\bar{c})\left(\lambda^{2}-\lambda-2\right)\right)}{2+4 \beta-8 \gamma+\left(1-12 \gamma-4 \beta^{2}\right) \lambda+4 \gamma \lambda^{3}}, \\
& x_{G_{3}}(\text { firm } 1)=\frac{(a-\bar{c})\left(\beta^{2} \lambda-\beta \lambda-2 \beta+2 \gamma \lambda^{3}-6 \gamma \lambda^{2}+8 \gamma+2\right)}{8 \gamma^{2} \lambda^{5}-8 \gamma^{2} \lambda^{4}-S_{1} \lambda^{3}+S_{2} \lambda^{2}+S_{3} \lambda+2\left(16 \gamma^{2}-4(\beta+2) \gamma+\beta-1\right)} \text {, } \\
& q_{G_{3}}(\text { firm } 1)=\frac{\left(2 \gamma(a-\bar{c})(\lambda+1)\left(\beta^{2} \lambda-\beta \lambda-2 \beta+2 \gamma \lambda^{3}-6 \gamma \lambda^{2}+8 \gamma+2\right)\right)}{8 \gamma^{2} \lambda^{5}-8 \gamma^{2} \lambda^{4}-S_{1} \lambda^{3}+S_{2} \lambda^{2}+S_{3} \lambda+2\left(16 \gamma^{2}-4(\beta+2) \gamma+\beta-1\right)} \text {, } \\
& x_{G_{3}}(\text { firm } 2)=\frac{(2 \gamma(\beta \lambda-2)(\lambda+1)(\lambda-2))(a-\bar{c})}{8 \gamma^{2} \lambda^{5}-8 \gamma^{2} \lambda^{4}-S_{1} \lambda^{3}+S_{2} \lambda^{2}+S_{3} \lambda+2\left(16 \gamma^{2}-4(\beta+2) \gamma+\beta-1\right)} \text {, } \\
& q_{G_{3}}(\text { firm } 2)=\frac{4 \gamma^{2}(a-\bar{c})\left(\lambda-\lambda^{2}+2\right)^{2}}{8 \gamma^{2} \lambda^{5}-8 \gamma^{2} \lambda^{4}-S_{1} \lambda^{3}+S_{2} \lambda^{2}+S_{3} \lambda+2\left(16 \gamma^{2}-4(\beta+2) \gamma+\beta-1\right)} \text {, } \\
& x_{G_{4}}(\text { firm } 1)=\frac{(\beta \lambda-2)(a-\bar{c})\left(2 \beta^{2} \lambda-3 \beta \lambda-2 \beta-2 \gamma \lambda^{3}+6 \gamma \lambda^{2}+\lambda-8 \gamma+2\right)}{2\left(-4 \gamma^{2} \lambda^{6}+12 \gamma^{2} \lambda^{5}+S_{4} \lambda^{4}+S_{5} \lambda^{3}+S_{6} \lambda^{2}+S_{7} \lambda+4\left(8 \gamma^{2}-6 \gamma-\beta^{2}+1\right)\right)} \text {, } \\
& q_{G_{4}}(\text { firm } 1)=\frac{\left(\gamma(a-\bar{c})\left(\lambda-\lambda^{2}+2\right)\left(3 \beta \lambda-2 \beta^{2} \lambda+2 \beta+2 \gamma \lambda^{3}-6 \gamma \lambda^{2}-\lambda+8 \gamma-2\right)\right)}{-4 \gamma^{2} \lambda^{6}+12 \gamma^{2} \lambda^{5}+S_{4} \lambda^{4}+S_{5} \lambda^{3}+S_{6} \lambda^{2}+S_{7} \lambda+4\left(8 \gamma^{2}-6 \gamma-\beta^{2}+1\right)}, \\
& x_{G_{4}}(\text { firm } 3)=\frac{(a-\bar{c})(\lambda-2 \beta \lambda+2)\left(\beta \lambda-\beta^{2} \lambda+2 \beta+\gamma \lambda^{3}-3 \gamma \lambda^{2}+4 \gamma-2\right)}{-4 \gamma^{2} \lambda^{6}+12 \gamma^{2} \lambda^{5}+S_{4} \lambda^{4}+S_{5} \lambda^{3}+S_{6} \lambda^{2}+S_{7} \lambda+4\left(8 \gamma^{2}-6 \gamma-\beta^{2}+1\right)} \text {, } \\
& q_{G_{4}}(\text { firm } 3)=\frac{\left(2 \gamma(a-\bar{c})\left(\lambda-\lambda^{2}+2\right)\left(\beta \lambda-\beta^{2} \lambda+2 \beta+\gamma \lambda^{3}-3 \gamma \lambda^{2}+4 \gamma-2\right)\right)}{-4 \gamma^{2} \lambda^{6}+12 \gamma^{2} \lambda^{5}+S_{4} \lambda^{4}+S_{5} \lambda^{3}+S_{6} \lambda^{2}+S_{7} \lambda+4\left(8 \gamma^{2}-6 \gamma-\beta^{2}+1\right)} \text {, }
\end{aligned}
$$

where $S_{1}=2\left(20 \gamma^{2}+(2 \beta+1) \gamma\right), S_{2}=2\left(4 \gamma^{2}+\left(2 \beta^{2}+7\right) \gamma\right), S_{3}=64 \gamma^{2}+4 \beta(\beta-1)(4 \gamma-1)$, $S_{4}=12 \gamma^{2}+\left(6 \beta^{2}-4 \beta+1\right) \gamma, S_{5}=-44 \gamma^{2}-\left(6 \beta^{2}+12 \beta-3\right) \gamma, S_{6}=\left(6+24 \beta-12 \beta^{2}\right) \gamma-24 \gamma^{2}-$ $\beta\left(\beta^{2}-1\right)(2 \beta-1), S_{7}=2\left(\beta\left(3 \beta^{2}-\beta-3\right)+24 \gamma^{2}-(10-16 \beta) \gamma+1\right)$.

\section{Acknowledgements}

This research was supported by King Saud University, Deanship of Scientific Research, College of Science Research Center.

Funding

This research was supported by King Saud University, Deanship of Scientific Research, College of Science Research Center.

Competing interests

The authors declare that they have no competing interests.

Authors' contributions

All authors read and approved the final manuscript. 


\section{Endnotes}

a Goyal and Moraga-Gonzalez [3] developed a theory of R\&D cooperation by involving the network concept.

b When we say size of the network, we mean the number of firms in the network.

C Goyal and Moraga-Gonzalez introduced network concepts to D'Aspremont and Jacquemin model for RJV competition (consistent with RJV competition given by [16]).

d Recall, if we consider asymmetric networks with the R\&D spillover $\beta$, there is a small possibility to find other stable networks in that area. For example, Goyal and Moraga-Gonzalez found that the low density network $G_{3}$ is stable for small values of the R\&D spillover (see Figure 4 for the list of asymmetric networks with three firms).

e Regarding to equation (11), see the equilibria under the symmetric networks in the Appendix.

\section{Publisher's Note}

Springer Nature remains neutral with regard to jurisdictional claims in published maps and institutional affiliations.

Received: 19 December 2016 Accepted: 19 July 2017 Published online: 01 August 2017

\section{References}

1. Deroian F. Dissemination of spillovers in cost-reducing alliances. Res Econ. 2008;62:34-44.

2. Goyal S, Joshi S. Networks of collaboration in oligopoly. Games Econ Behav. 2003;43(1):57-85.

3. Goyal S, Moraga-Gonzalez JL. R\&D networks. Rand J Econ. 2001;32:686-707.

4. Song H, Vannetelbosch V. International R\&D collaboration networks. Manch Sch. 2007;75(6):742-66.

5. Jackson M. Social and economic networks. Princeton: Princeton University Press; 2008.

6. Newman MEJ. The structure and function of complex networks. SIAM Rev. 2003;45:167-256.

7. Hackner J. A note on price and quantity competition in differentiated oligopolies. J Econ Theory. 2000;93:233-9.

8. Konig MD, Battiston S, Napoletano M, Schweitzer F. The efficiency and stability of R\&D networks. Games Econ Behav. 2012;75:694-713.

9. D'Aspremont C, Jacquemin A. Cooperative and noncooperative R\&D in duopoly with spillovers. Am Econ Rev. 1988;78:1133-7.

10. Jackson MO, Wolinsky A. A strategic model of social and economic networks. J Econ Theory. 1996;71:44-74

11. Alghamdi M. Economic returns in forming stable R\&D networks. SpringerPlus. 2016;5(1):1570.

12. Ahuja G. Collaboration networks, structural holes, and innovation: a longitudinal study. Adm Sci Q. 2000;45:425-55.

13. Stuart TE. Interorganizational alliances and the performance of firms: a study of growth and innovation rates in a high-technology industry. Strateg Manag J. 2000;21:791-811.

14. Tomasello MV, Napoletano M, Garasz A, Schweitzer F. The rise and fall of R\&D networks. 2013. arXiv:1304.3623v1.

15. Verspagen B, Duysters $G$. The small worlds of strategic technology alliances. Technovation. 2004;24:563-71.

16. Kamien M, Muller E, Zang I. Research joint ventures and R\&D cartels. Am Econ Rev. 1992;82:1293-306.

\section{Submit your manuscript to a SpringerOpen ${ }^{\circ}$ journal and benefit from:}

- Convenient online submission

- Rigorous peer review

- Open access: articles freely available online

- High visibility within the field

- Retaining the copyright to your article

Submit your next manuscript at $\gg$ springeropen.com 